\title{
CRITICAL ASSOCIATED METRICS ON CONTACT MANIFOLDS. II
}

\author{
D. E. BLAIR and A. J. LEDGER
}

(Received 18 February 1985)

Communicated by K. Mackenzie

\begin{abstract}
The study of the integral of the scalar curvature, $\int_{M} R d V_{g}$, as a function on the set of all Riemannian metrics of the same total volume on a compact manifold is now classical, and the critical points are the Einstein metrics. On a compact contact manifold we consider this and $\int_{M}\left(R-R^{*}-4 n^{2}\right) d V$, with $R^{*}$ the *-scalar curvature, as functions on the set of metrics associated to the contact structure. For these integrals the critical point conditions then become certain commutativity conditions on the Ricci operator and the fundamental collineation of the contact metric structure. In particular, Sasakian metrics, when they exist, are maxima for the second function.
\end{abstract}

1980 Mathematics subject classification (Amer. Math. Soc.): 58 E 11, 53 C 15, 53 C 25.

\section{Introduction}

The study of the integral of the scalar curvature, $I(g)=\int_{M} R d V_{g}$, as a function on the set of all Riemannian metrics of the same total volume on a compact manifold is well known, and the critical points are the Einstein metrics (see e.g. [5]). Here we consider the function $I$ restricted to the set of metrics associated to a contact structure on a compact contact manifold. As this is a smaller set of metrics, one would expect a weaker critical point condition, and we shall show (Theorem 1) that the condition is that the Ricci operator $Q$ and the fundamental collineation $\phi$ when restricted to the contact distribution commute.

On a contact metric manifold we have

$$
R-R^{*}-4 n^{2}=-\frac{1}{2}\|\nabla \phi\|^{2}+2 n-\operatorname{tr} h^{2} \leqslant 0,
$$

(C) 1986 Australian Mathematical Society $0263-6115 / 86 \$ A 2.00+0.00$ 
where $R^{*}$ is the *-scalar curvature, and where $h=\frac{1}{2} \mathfrak{R}_{\xi} \phi$ (see Section 2 for definitions), with equality holding if and only if the structure is Sasakian [6]. This suggests the study of

$$
K(g)=\int_{M}\left(R-R^{*}-4 n^{2}\right) d V
$$

where Sasakian metrics, when they exist, are maxima. Here we shall show that the critical point condition is that $Q-2 n h$ and $\phi$ commute when restricted to the contact distribution.

Similar results to these for symplectic manifolds can be found in [4]; there the critical point condition for both $I$ and $K$ is $J Q=Q J$, with $J$ the corresponding almost complex structure.

Again in the contact case the integral of the Ricci curvature in the direction of the characteristic vector field $\xi$ was studied in [3]. On a regular contact manifold the critical points were shown to be the $K$-contact metrics, i.e. the associated metrics for which $\xi$ generates a 1-parameter group of isometries.

\section{Preliminaries.}

By a contact manifold we mean a $(2 n+1)$-dimensional $C^{\infty}$ manifold $M$ together with a global 1-form $\eta$ such that $\eta \wedge(d \eta)^{n} \neq 0$. Given a contact form $\eta$, it is well known that there exists a unique vector field $\xi$, called the characteristic vector field of $\eta$, satisfying $\eta(\xi)=1$ and $d \eta(\xi, X)=0$ for all vector fields $X$. A Riemannian metric $g$ is said to be an associated metric if there exists a tensor field $\phi$ of type $(1,1)$ such that $d \eta(X, Y)=g(X, \phi Y), \eta(X)=g(X, \xi)$ and $\phi^{2}=-I+\eta \otimes \xi$. These metrics are constructed by the polarization of $d \eta$ evaluated on a local orthonormal basis of an arbitrary metric on the contact distribution (subbundle) $\{\eta=0\}$; see [1] as a general reference or e.g. [2]. The set $\mathscr{A}$ of all associated metrics is infinite dimensional and is totally geodesic in the set of all Riemannian metrics on $M[2,3]$. We also refer to the pair $(\eta, g)$ or to $(\phi, \xi, \eta, g)$ as a contact metric structure. Finally note that all metrics associated to $\eta$ have the same volume element, viz. a constant depending on $n$ times $\eta \wedge(d \eta)^{n}$ (see e.g. [7]), and hence we will write $d V$ instead of $d V_{g}$.

Given a contact metric structure $(\phi, \xi, \eta, g)$, define a tensor field $h$ by $h=\frac{1}{2} \mathfrak{L}_{\xi} \phi$ where $\mathfrak{Q}$ denotes Lie differentiation. Then $h$ is a symmetric operator which anticommutes with $\phi ; h \xi=0 ; h$ vanishes if and only if $\xi$ is Killing; and $h$ is related to the covariant derivative of $\xi$ by

$$
\nabla_{i} \xi^{j}=\phi_{i}{ }^{j}+h_{i}{ }^{m} \phi_{m}{ }^{j}
$$

[1]. Other formulas for a general contact metric structure that we will need are

$$
\nabla, \phi_{j}{ }^{t}=2 n \eta_{j},
$$




$$
\left(\nabla_{k} \phi_{i p}\right) \phi_{j}^{p}=\phi_{k}^{p} \nabla_{p} \phi_{i j}+\eta_{j} \phi_{k i}-\eta_{j} h_{k m} \phi^{m}{ }_{i}+2 \phi_{j k} \eta_{i}
$$

[6].

We now give two definitions on a contact metric manifold and derive a further identity. The *-Ricci tensor and the *-scalar curvature are defined by

$$
R_{i j}^{*}=R_{i k l t} \phi^{k l} \phi_{j}{ }^{\prime}, R^{*}=R_{i}^{* i} .
$$

Contracting the Ricci identity

$$
\nabla_{t} \nabla_{k} \phi_{j}{ }^{s}-\nabla_{k} \nabla_{t} \phi_{j}{ }^{s}=R_{t k p}{ }^{s} \phi_{j}^{p}-R_{t k j}{ }^{p} \phi_{p}{ }^{s}
$$

and using equation (2.2) we have

$$
\nabla_{t} \nabla_{k} \phi_{j}{ }^{t}=R_{k p} \phi_{j}^{p}+R_{k t p j} \phi^{t p}+2 n \nabla_{k} \eta_{j}
$$

Now using equation (2.1) and symmetrizing we obtain

$$
\nabla_{t} \nabla_{k} \phi_{j}{ }^{t}+\nabla_{t} \nabla_{j} \phi_{k}{ }^{t}=R_{k t} \phi_{j}{ }^{t}+R_{j t} \phi_{k}{ }^{t}+2 n\left(h_{k m} \phi_{j}^{m}+h_{j m} \phi^{m}{ }_{k}\right) .
$$

\section{Critical associated metrics}

Let $g(t)$ be a smooth curve in $\mathscr{A}$ and let

$$
D_{i j}=\left.\frac{\partial g_{i j}}{\partial t}\right|_{t=0} .
$$

We also write $D$ for the tensor field of type $(1,1)$ corresponding to $D_{i j}$ via $g=g(0)$ and let $\phi$ be the fundamental collineation corresponding to $g$. Then since $D$ is the tangent to the path $g(t)$ in $\mathscr{A}$ we have

$$
D \phi+\phi D=0, \quad D \xi=0
$$

$[2,3]$.

Lemma. Let $T$ be a second order symmetric tensor field on $M$. Then

$$
\int_{M} T^{i j} D_{i j} d V=0
$$

for all $D$ satisfying (3.1) if and only if $T$ and $\phi$ commute when restricted to the contact distribution, $\{\eta=0\}$, i.e. for $X \in\{\eta=0\}$, the projection of $(\phi T-T \phi) X$ to $\{\boldsymbol{\eta}=0\}$ vanishes; equivalently

$$
T_{i j}=T_{p q} \phi_{i}^{p} \phi_{j}^{q}+T_{j r} \xi^{r} \eta_{i}+T_{i r} \xi^{r} \eta_{j}-\left(T_{r s} \xi^{r} \xi^{s}\right) \eta_{i} \eta_{j}
$$

Proof. Let $X_{1}, \ldots, X_{2 n}, \xi$ be a local $\phi$-basis defined on a neighborhood $U$ (i.e. $X_{1}, \ldots, X_{2 n}, \xi$ is orthonormal with respect to $g$ and $\left.X_{2 i}=\phi X_{2 i-1}\right)$ and note that for $X_{1}$ we can take any unit vector field orthogonal to $\xi$. Let $f$ be a $C^{\infty}$ function with compact support in $U$ and consider the 2-dimensional distribution over $U$ 
determined by $X_{1}$ and $X_{2}$. Define a curve $g(t)$ of metrics by the deformation on this distribution given by the matrix

$$
\left(\begin{array}{cc}
1+t f+\frac{1}{2} t^{2} f^{2} & \frac{1}{2} t^{2} f^{2} \\
\frac{1}{2} t^{2} f^{2} & 1-t f+\frac{1}{2} t^{2} f^{2}
\end{array}\right)
$$

with no change in $g$ elsewhere or in other directions. It is easy to check that $g(t)$ is a curve in $\mathscr{A}$, and clearly the only non-zero components of $D$ are $D_{11}=-D_{22}$ $=f$. Then (3.2) becomes

$$
\int_{M}\left(T^{11}-T^{22}\right) f d V=0
$$

from which, since $f$ is any $C^{\infty}$ function with compact support in $U$ and $X_{1}$ is any unit vector field orthogonal to $\xi$, we obtain

$$
T(X, X)=T(\phi X, \phi X)
$$

for any vector field $X$ orthogonal to $\xi$. Since $T$ is symmetric, we have

$$
T(X, Y)=T(\phi X, \phi Y)
$$

for $X, Y \in\{\eta=0\}$. Now replacing $X$ and $Y$ by $\phi^{2} X$ and $\phi^{2} Y$ with $X$ and $Y$ arbitrary vector fields, and noting that $\phi^{3}=-\phi$, we have

$$
\begin{aligned}
T(\phi X, \phi Y) & =T\left(\phi^{2} X, \phi^{2} Y\right) \\
& =T(-X+\eta(X) \xi,-Y+\eta(Y) \xi) \\
& =T(X, Y)-\eta(X) T(\xi, Y)-\eta(Y) T(\xi, X)+T(\xi, \xi) \eta(X) \eta(Y)
\end{aligned}
$$

as desired; note also that this is equivalent to

$$
\phi T-T \phi=\eta \otimes \phi T \xi-(\eta \circ T \phi) \otimes \xi .
$$

Conversely, if $T$ satisfies (3.4) and $D$ satisfies (3.1), then

$$
\operatorname{tr} T D=\operatorname{tr} T \phi D \phi=\operatorname{tr} \phi T D \phi=-\operatorname{tr} T D,
$$

which implies that $T^{i j} D_{i j}=0$.

Since $d \eta$ is the covariant form of $\phi$, and since the contact structure is fixed, we have $\partial \phi_{k l} / \partial t=0$, and since $\partial g^{i j} /\left.\partial t\right|_{t=0}=-D^{i j}$, we have $\partial \phi_{j}^{i} /\left.\partial t\right|_{t=0}=-D^{i m} \phi_{m j}$. Similarly, using (3.1), we obtain $\partial \phi^{i j} /\left.\partial t\right|_{t=0}=0$. Finally, we define two other tensor fields

$$
D_{j i}^{h}=\frac{1}{2}\left(\nabla_{j} D_{i}^{h}+\nabla_{i} D_{j}^{h}-\nabla^{h} D_{i j}\right)
$$

and

$$
D_{k j i}{ }^{h}=\nabla_{k} D_{j i}{ }^{h}-\nabla_{j} D_{k i}{ }^{h},
$$

where $\nabla$ denotes the Riemannian connection of $g(0)$. Then

$$
D_{k j i}{ }^{h}=\left.\frac{\partial R_{k j i}{ }^{h}}{\partial t}\right|_{t=0}
$$

(see, for example [5]). 
THEOREM 1. Let $M$ be a compact contact manifold and $\mathscr{A}$ the set of metrics associated to the contact form. Then a metric $g \in \mathscr{A}$ is a critical point of the function $I(g)=\int_{M} R d V$ if and only if the Ricci operator and $\phi$ commute when restricted to the contact distribution.

Proof. The proof involves setting $d I /\left.d t\right|_{t=0}=0$ for a path $g(t)$ in $\mathscr{A}$ with $g(0)=g$. First we have

$$
\frac{d}{d t} \int_{M} R d V=\int_{M} \frac{d}{d t}\left(R_{k j i}{ }^{k} g^{j i}\right) d V=\int_{M}\left(D_{k j i}{ }^{k} g^{j i}-R_{j i} D^{j i}\right) d V .
$$

From (3.1) it is immediate that $\operatorname{tr} D=0$, and hence $D_{k i}{ }^{k}=0$. Therefore,

$$
D_{k j i}{ }^{k}{ }^{j i}=\left(\nabla_{k} D_{j i}{ }^{k}\right) g^{j i}=\nabla_{k}\left(D_{j i}{ }^{k} g^{j i}\right) \text {, }
$$

which is a divergence. Hence

$$
\left.\frac{d I}{d t}\right|_{t=0}=-\int_{M} R^{j i} D_{j i} d V
$$

and the result follows from the lemma.

THEOREM 2. Let $M$ be a compact contact manifold of dimension $2 n+1$ and $\mathscr{A}$ the set of metrics associated to the contact form. Then a metric $g \in \mathscr{A}$ is a critical point of the function $K(g)=\int_{M}\left(R-R^{*}-4 n^{2}\right) d V$ if and only if the operators $Q-2$ nh and $\phi$ commute when restricted to the contact distribution.

Proof. We begin by computing $d R^{*} / d t$, bearing in mind the basic properties of the structure tensors, especially the following in approximately the order listed: skew-symmetry of $\phi$, symmetry of $D_{k} l^{\prime}$, symmetry of $D_{i j},(2.2),(2.1),(3.1)$, $\operatorname{tr} D=0$, and $\phi h+h \phi=0$.

$$
\begin{aligned}
\frac{d R^{*}}{d t} & =\frac{d}{d t} R_{i k l}{ }^{j} \phi^{k l} \phi_{j}^{i} \\
& =D_{i k l}{ }_{j}{ }^{k l} \phi_{j}^{i}-R_{i k l}{ }^{j} \phi^{k l} \phi_{m j} D^{i m} \\
& =-\frac{1}{2}\left(\nabla_{k}\left(\nabla_{i} D_{l}^{j}+\nabla_{l} D_{i}^{j}-\nabla^{j} D_{i l}\right)\right) \phi^{k l} \phi_{j}^{i}-R_{i m}^{*} D^{i m} \\
& =-\left(\nabla_{k} \nabla_{i} D_{l}^{j}\right) \phi^{k l} \phi_{j}^{i}-R_{i m}^{*} D^{i m} \\
& =\operatorname{div}+\left(\nabla_{i} D_{l}^{j}\right)\left(\nabla_{k} \phi^{k l} \phi_{j}^{i}\right)-R_{i m}^{*} D^{i m} \\
& =\operatorname{div}-D_{l}^{j} \nabla_{i}\left(-2 n \xi^{l} \phi_{j}^{i}+\phi^{k l} \nabla_{k} \phi_{j}^{i}\right)-R_{i m}^{*} D^{i m} \\
& =\operatorname{div}+2 n D_{l}^{j}\left(\phi_{i}{ }^{l}+h_{i m} \phi^{m l}\right) \phi_{j}^{i}-D_{l}^{j} \nabla_{i}\left(\phi^{k l} \nabla_{k} \phi_{j}^{i}\right)-R_{i m}^{*} D^{i m} \\
& =\operatorname{div}-2 n D_{l}^{j} h_{j}^{l}-D_{l}^{j} \nabla_{i}\left(\phi^{k l} \nabla_{k} \phi_{j}^{i}\right)-R^{* j l} D_{j l},
\end{aligned}
$$


where div denotes terms which are divergences and hence which contribute nothing to the integral. Now combining this with the proof of Theorem 1, we have

$$
\left.\frac{d K}{d t}\right|_{t=0}=\int_{M}\left[-R^{j l}+2 n h^{j l}+\nabla_{i}\left(\phi^{k l} \nabla_{k} \phi^{i j}\right)+R^{* j l}\right] D_{j l} d V .
$$

From the lemma we see that the condition for a critical point is that the symmetric part of the expression in square brackets satisfies equation (3.3); i.e. noting that $h^{\prime r} \eta_{r}=0$ and that $R^{* l r} \eta_{r}=0$, we have

$$
\begin{aligned}
\text { (3.5) }-R^{j l}+ & 2 n h^{j l}+\frac{1}{2} \nabla_{i}\left(\phi^{k l} \nabla_{k} \phi^{i j}+\phi^{k j} \nabla_{k} \phi^{i l}\right)+\frac{1}{2}\left(R^{* j l}+R^{* l j}\right) \\
= & -R^{p q} \phi_{p}^{j} \phi_{q}^{l}+2 n h^{p q} \phi^{j}{ }_{\phi^{\prime}}{ }_{q} \\
& +\frac{1}{2}\left(\nabla_{i}\left(\phi^{k q} \nabla_{k} \phi^{i p}+\phi^{k p} \nabla_{k} \phi^{i q}\right)\right) \phi^{j}{ }_{p} \phi_{q}^{\prime}+\frac{1}{2}\left(R^{* p q}+R^{* q p}\right) \phi^{j}{ }_{p} \phi_{q}^{\prime} \\
& +\xi^{l} \eta_{r}\left(-R^{j r}+\frac{1}{2} \nabla_{i}\left(\phi^{k r} \nabla_{k} \phi^{i j}+\phi^{k j} \nabla_{k} \phi^{i r}\right)+\frac{1}{2} R^{* r j}\right) \\
& +\xi^{j} \eta_{r}\left(-R^{i r}+\frac{1}{2} \nabla_{i}\left(\phi^{k r} \nabla_{k} \phi^{i l}+\phi^{k l} \nabla_{k} \phi^{i r}\right)+\frac{1}{2} R^{* r l}\right) \\
& -\xi^{j} \xi^{l}\left(-R^{r s} \eta_{r} \eta_{s}+\frac{1}{2}\left(\nabla_{i}\left(\phi^{k s} \nabla_{k} \phi^{i r}+\phi^{k r} \nabla_{k} \phi^{i s}\right)\right) \eta_{r} \eta_{s}\right) .
\end{aligned}
$$

From the definition of $R_{j l}^{*}$ it is easy to see that all terms involving the *-Ricci tensor vanish. Turning to the terms involving covariant derivatives, we first obtain on the left an expression of the form

$$
\frac{1}{2}\left(\left(\nabla_{i} \phi^{k l}\right)\left(\nabla_{k} \phi^{i j}\right)+\left(\nabla_{i} \phi^{k j}\right)\left(\nabla_{k} \phi^{i l}\right)\right) .
$$

To the similar term of the right we apply (2.3) as well in order to show separately that

$$
\xi^{j} \eta_{r}\left(\nabla_{i} \phi^{k l}\right)\left(\nabla_{k} \phi^{i r}\right)=2 n \xi^{j} \xi^{l}+\left(\nabla_{i} \phi_{q}^{l}\right) h^{q i} \xi^{j}
$$

mainly by virtue of (2.1), and that

$$
-\xi^{j} \xi^{\prime} \eta_{r} \eta_{s}\left(\nabla_{i} \phi^{k s}\right)\left(\nabla_{k} \phi^{i r}\right)=-\left(4 n-R_{i k} \xi^{i} \xi^{k}\right) \xi^{j} \xi^{l},
$$

again by virtue of (2.1) and by the fact that $R_{i k} \xi^{i} \xi^{k}=2 n-\operatorname{tr} h^{2}$ [1, p. 67]. Then the terms involving products of first derivatives all cancel. For the second derivative terms, compare $\frac{1}{2} \phi^{k l} \nabla_{i} \nabla_{k} \phi^{i j}$ on the left with $\frac{1}{2} \phi^{k p}\left(\nabla_{i} \nabla_{k} \phi^{i q}\right) \phi_{p}^{j} \phi_{q}^{\prime}$ on the right and apply (2.4); similarly apply (2.4) to groupings of the other second derivative terms. The final result is that (3.5) becomes

$$
\begin{aligned}
-2 R^{j l} & +4 n h^{j l} \\
& =-2 R^{p q} \phi_{p}^{j} \phi_{q}^{l}+4 n h^{p q} \phi_{p}^{j} \phi_{q}^{l}-2 R_{r}^{j} \xi^{r} \xi^{l}-2 R_{r}^{\prime} \xi^{r} \xi^{j}+2\left(R_{r s} \xi^{r} \xi^{s}\right) \xi^{j} \xi^{l}
\end{aligned}
$$

which, since $h \xi=0$, completes the proof.

Combining Theorems 1 and 2 we have the following result.

COROLLARY. If $g$ is a critical point of both $I$ and $K$, then $\xi$ is a Killing vector field with respect to $g$, i.e. $(\phi, \xi, \eta, g)$ is a $K$-contact structure. If $(\phi, \xi, \eta, g)$ is Sasakian, then $g$ is a critical point of both $I$ and $K$. 
PROOF. If $g$ is a critical point of $I$ and $K$, then from the theorems both $R_{i j}$ and $R_{i j}-2 n h_{i j}$ satisfy equation (3.3). Thus, since $h \xi=0$, we have $h_{i j}=h_{p q} \phi_{i}{ }^{p} \phi_{j}{ }^{q}$, but since $\phi$ and $h$ anti-commute, it follows that $h=0$, which implies that $\xi$ is Killing. If $(\phi, \xi, \eta, g)$ is Sasakian, then it is automatically $K$-contact, so that $h=0$, and, moreover, $Q \phi=\phi Q[1, \mathrm{p} .76]$. This gives the result.

In general a $K$-contact structure need not satisfy $Q \phi=\phi Q$, and it is an open question as to whether or not a $K$-contact structure satisfying $Q \phi=\phi Q$ is Sasakian. We also remark that a contact metric structure may satisfy $Q \phi=\phi Q$ without being $K$-contact; e.g. the usual contact structure on the 3-dimensional torus carries a flat associated metric which is not $K$-contact [1, p. 123], and so this metric is critical for $I$ but not for $K$.

\section{References}

[1] D. E. Blair, Contact manifolds in Riemannian geometry (Lecture Notes in Mathematics, Vol. 509, Springer, Berlin, 1976).

[2] D. E. Blair, 'On the set of metrics associated to a symplectic or contact form', Bull. Inst. Math. Acad. Sinica 11 (1983), 297-308.

[3] D. E. Blair, 'Critical associated metrics on contact manifolds', J. Austral. Math. Soc. (Series A) 37 (1984), $82-88$

[4] D. E. Blair and S. Ianus, 'Critical associated metrics on symplectic manifolds', Contemp. Math., to appear.

[5] Y. Muto, 'On Einstein metrics', J. Differential Geom. 9 (1974) 521-530.

[6] Z. Olszak, 'On contact metric manifolds', Tôhoku Math. J. 31 (1979), 247-253.

[7] S. Sasaki, Almost contact manifolds (Lecture Notes, Mathematical Institute, T'ôhoku University, Vol. 1, 1965).

Department of Mathematics

Michigan State University

East Lansing, Michigan 48824

U.S.A.
Department of Pure Mathematics University of Liverpool Liverpool L69 3BX United Kingdom 